Pacific Journal of Mathematics

ON THE MINIMAL DISPLACEMENT OF POINTS UNDER

Kazingez Goober 


\title{
ON THE MINIMAL DISPLACEMENT OF POINTS UNDER LIPSCHITZIAN MAPPINGS
}

\author{
K. GOEBEL
}

The aim of this paper is to study the evaluation of the quantity inf $\|x-T x\|$ when $T$ is a Lipschitzian self mapping of a closed bounded and convex subset of a Banach space. It is proved that in an arbitrary Banach space there exists a function $\varphi(k):\langle 1, \infty) \rightarrow\langle 0,1)$ such that for arbitrary $T: X \rightarrow X$ satisfying a Lipschitz condition constant $k>1$, inf $\|x-T x\| \leqq$ $\varphi(k) r(X)$ where $r(X)$ denotes the radius of the set $X$. Some precise formulas for $\varphi(k)$ are obtained in certain spaces along with some general evaluations of it in arbitrary spaces. In particular, the casc of Hilbert space is considered and some evaluations for $\varphi(k)$ are obtained in that setting.

Introduction. According to the well known Brouwer's fixed point theorem, an arbitrary continuous self mapping of a bounded closed and convex subset of a finite dimensional Banach space has a fixed point. This result is not true in arbitrary infinite dimensional spaces even for nonexpansive mappings. F. E. Browder [1], D. Göhde [3], and W. A. Kirk [4] independently proved that the fixed point property does hold for nonexpansive mapping under some additional assumptions concerning the properties of the space (e.g., uniform convexity, normal structure), and in Kirk's paper [4] there is an example showing that this result cannot be extended to the case of a mapping with Lipschitz constant $k>1$. However, most of the quoted examples of fixed point free continuous self mappings of bounded closed and convex sets in Banach spaces have the property that

$$
\inf \|x-T x\|=0 \text {. }
$$

In particular, all nonexpansive mappings have to have this property.

We shall deal here with the problem of evaluation of the quantity $\|x-T x\|$ where $T$ lies in the class of Lipschitzian mappings with constant $k$. This problem will be called "minimum distance problem."

In this paper we show that for any Banach space $B$ there exists a function $\varphi_{B}(k):\langle 1, \infty) \rightarrow\langle 0,1)$ such that for arbitrary closed bounded and convex sets $x \subset B$ and arbitrary $T: X \rightarrow X$ satisfying a Lipschitz condition with constant $k \geqq 1$,

$$
\inf \|x-T x\| \leqq \varphi(k) r(X)
$$

where $r(X)$ is the radius of the set $X$.

Some precise formulas for $\varphi(k)$ are obtained in certain spaces 
(e.g., $C, L^{1}, c_{0}$ ) along with some general evaluations of it in arbitrary spaces. We also consider the function $\varphi(k)$ in Hilbert space and determine some of its properties there.

We feel that an important part of this paper is the last section in which some, in our opinion, interesting and seemingly difficult questions are raised.

Notations and definitions. Let $B$ denote an arbitrary Banach space with the norm \|\| and zero element $\theta . \mathfrak{M}_{B}$ will denote the family of all nonempty, closed, bounded and convex subsets of $B$. Sets belonging to $\mathfrak{M}_{B}$ will be denoted by principal letters $X, Y, Z, \cdots$ and elements of $B$ by $x, y, z, \cdots, K(x, r)$ will denote the closed ball centered at $x$ with radius $r$ and $S(x, r)$ will be its boundary. $K(\theta, r)$ and $S(\theta, r)$ will be shortly denoted $K_{r}$ and $S_{r}$. For arbitrary set $X$, let $d(X)$ and Conv $X$ denote the diameter of $X$ and the convex closure of $X$, respectively.

For arbitrary $X \in \mathfrak{M}_{B}$ and $x \in X$ put

$$
\begin{aligned}
r(x, X) & =\sup [\|x-y\|: y \in X] \\
r(X) & =\inf [r(x, X): x \in X] \\
C(X) & =[x: r(x, X)=r(X)] .
\end{aligned}
$$

The numbers $r(x, X), r(X)$ will be called the radius of $X$ with respect to $x$ and the radius of $X$, respectively. $C(X)$ will be called the center of $X$. It is well known [5] that in reflexive spaces $C(X)$ is a nonempty closed and convex set, and if $B$ is uniformly convex it consists of only one point.

For an arbitrary set $X \in \mathfrak{M}_{B}$ we shall consider the family $\mathscr{L}(X)$ of all transformations of $X$ into itself satisfying the Lipschitz condition

$$
\|T x-T y\| \leqq k\|k-y\|,
$$

for some constant $k$. The subfamily of $\mathscr{L}(X)$ consisting of all mappings satisfying (1) with fixed costant $k$ will be denoted $\mathscr{L}(k, X)$. It is obvious that $k_{1} \leqq k_{2}$ implies $\mathscr{L}\left(k_{1}, X\right) \subset \mathscr{L}\left(k_{2}, X\right)$ and that

$$
\mathscr{L}(X)=\bigcup_{k>0} \mathscr{L}(k, X) \text {. }
$$

Moreover, if $T_{1} \in \mathscr{L}\left(k_{1} X\right), T_{2} \in \mathscr{L}\left(k_{2}, X\right)$ and $\alpha \in\langle 0,1\rangle$ then $T=\alpha T_{1}+$ $(1-\alpha) T_{2} \in \mathscr{L}\left(\alpha k_{1}+(1-\alpha) k_{2}\right)$.

The mapping $T$ is said to be nonexpansive if $T \in \mathscr{L}(1, k)$ and $T$ is said to be a contraction if $T \in \mathscr{L}(k, X)$ with $k<1$.

Statement of the main problem. Let $X \in \mathfrak{M}_{B}$ and let $T \in \mathscr{L}(k, X)$, 
$k \geqq 1$. We shall start with the following:

THEOREM 1. $\inf [\|x-T x\|: x \in X] \leqq r(X)\left(1-\frac{1}{k}\right)$.

Proof. Let $y \in X$ be such that $r(y, X) \leqq r(X)+\varepsilon$. Put

$$
T^{*} x=\left(1-\frac{1}{k+\varepsilon}\right) y+\frac{1}{k+\varepsilon} T x .
$$

Then $T^{*} \in \mathscr{L}(k /(k+\varepsilon), X)$ and by Banach contraction principle it has fixed point $x^{*}$. Now we have

$$
\begin{aligned}
\left\|x^{*}-T x^{*}\right\| & =\left\|\left(1-\frac{1}{k+\varepsilon}\right) y-\left(1-\frac{1}{k+\varepsilon}\right) T x^{*}\right\| \leqq\left(1-\frac{1}{k+\varepsilon}\right)\left\|y-T x^{*}\right\| \\
& \leqq(r(X)+\varepsilon)\left(1-\frac{1}{k+\varepsilon}\right)
\end{aligned}
$$

from which the theorem follows.

We now introduce some functions which characterize our problem. Put

$$
\varphi^{*}(k, X)=\sup _{T \in \mathscr{L}(k, X)} \inf [\|x-T x\|: x \in X] .
$$

In view of Theorem 1

$$
\varphi^{*}(k, X) \leqq r(X)\left(1-\frac{1}{k}\right) .
$$

Notice that if $X, Y \in \mathfrak{M}_{B}$ are such that $X=u+\alpha Y$ where $u \in B$ and $\alpha$ is a number then $r(X)=|\alpha| r(Y)$ and each transformation $T \in$ $\mathscr{L}(k, X)$ determine the transformation

$$
T^{*} y=\frac{1}{\alpha}(T(u+\alpha y)-u)
$$

which belongs to $\mathscr{L}(k, Y)$. This implies that

$$
\varphi^{*}(k, X)=|\alpha| \varphi^{*}(k, Y)
$$

and for this reason it will be more convenient to consider the function

$$
\varphi(k, X)=\frac{\varphi^{*}(k, X)}{r(X)}
$$

Obviously

$$
\varphi(k, X) \leqq 1-\frac{1}{k},
$$


and if $X=u+\alpha Y$, then $\varphi(k, X)=\varphi(k, Y)$. Put

Finally we define a function which characterizes the whole space.

$$
\varphi_{B}(k)=\sup \left[\varphi(k, X): X \in \mathfrak{M}_{B}\right],
$$

or more precisely

$$
\varphi_{B}(k)=\sup \left\{\inf _{x \in X} \frac{\|x-T x\|}{r(X)}: X \in \mathfrak{M}_{B}, T \in \mathscr{L}(k, X)\right\} .
$$

The functions $\varphi(k, X)$ and $\varphi_{B}(k)$ will be called the minimal distance characteristic of $X$ and the minimal distance characteristic of the space $B$, respectively.

Obviously

$$
\varphi_{B}(k)=0 \text { for } 0 \leqq k \leqq 1
$$

and

$$
\varphi_{B}(k) \leqq 1-\frac{1}{k} \quad \text { for } k \geqq 1 \text {. }
$$

Moreover $\varphi_{B}(k)$ is nondecreasing and $\varphi_{B}(k) \equiv 0$ for finite dimensional B.

In the situations where it will not be misleading we shall write $\varphi(k)$ instead of $\varphi_{B}(k)$. Furthermore we shall always consider the function $\varphi(k)$ as defined only on the interval $\langle 1, \infty)$.

Three examples. Let us start by showing that in some spaces oue evaluation for $\varphi_{B}(k)$ is exact.

EXAMPLE 1. Let $X$ be the subset of $C\langle 0,1\rangle$ defined by the following formula,

$$
X=[x=\{x(t)\}: 0=x(0) \leqq x(t) \leqq x(1)=1] .
$$

It is easy to see that $X \in \mathfrak{M}_{C\langle 0,1\rangle}$ and $r(X)=1$. Put

$$
(T x)(t)=k \max \left[x(t)-1+\frac{1}{k}, 0\right] .
$$

This transformation belongs to $\mathscr{L}(k, X)$. For an arbitrary function $x \in X$, there exists $\bar{t} \in\langle 0,1\rangle$ such that $x(\bar{t})=1-1 / k$ so obviously $(T x)(\bar{t})=0$ and we have

$$
\|x-T x\| \geqq|x(\bar{t})-(T x)(\bar{t})|=1-\frac{1}{k},
$$

and this shows that 


$$
\varphi_{C<0,1\rangle}(k)=1-\frac{1}{k}
$$

for $k \geqq 1$.

Example 2. Let $X$ be a subset of $L^{1}(0,1)$ defined as follows

$$
X=\left[f \in L^{1}: 0 \leqq f(t),\|f\|_{L^{1}}=1\right] \text {. }
$$

Notice that $X \in \mathfrak{M}_{L^{1}}$ and $r(X)=2$.

For an arbitrary function $f \in X$, put

$$
t_{f}=\sup \left[t: \int_{0}^{t} f(s) d s=1-\frac{1}{k}\right]
$$

and

$$
(T f)(t)= \begin{cases}0 & \text { for } t \leqq t_{f} \\ k f(t) & \text { for } t>t_{f}\end{cases}
$$

Suppose now that $f, g \in X$ and $t_{f} \leqq t_{g}$. We have

$$
\begin{aligned}
\|T f-T g\| & =\int_{0}^{1}|(T f)(s)-(T g)(s)| d s=k \int_{t_{f}}^{t_{g}} f(s) d s+k \int_{t_{g}}^{1}|f(s)-g(s)| d s \\
& =k \int_{0}^{t_{g}} f(s) d s-k\left(1-\frac{1}{k}\right)+k \int_{t_{g}}^{1}|f(s)-g(s)| d s \\
& =k\left(\int_{0}^{t_{g}} f(s) d s-\int_{0}^{t_{g}} g(s) d s+\int_{t_{g}}^{1}|f(s)-g(s)| d s\right) \\
& \leqq k\|f-g\| .
\end{aligned}
$$

This shows that $T \in \mathscr{L}(k, X)$. On the other hand we have

$$
\begin{aligned}
\|f-T f\| & =\int_{0}^{1}|f(s)-(T f)(s)| d s=\int_{0}^{t_{f}} f(s) d s+\int_{t_{f}}^{1}(k-1) f(s) d s \\
& =2\left(1-\frac{1}{k}\right)=r(X)\left(1-\frac{1}{k}\right)
\end{aligned}
$$

and hence

$$
\varphi_{L^{1}(0,1)}(k)=1-\frac{1}{k} .
$$

Finally we show that the same is true in the space $c_{0}$.

ExAmple 3. Suppose $X$ is the subset of $c_{0}$ defined by

$$
X=\left[x=\left\{x_{i}\right\}: x_{1}=1,0 \leqq x_{i} \leqq 1\right] \text {. }
$$

Then $X \in \mathfrak{M}_{c_{0}}$ and $r(X)=1$. Put 


$$
(T x)_{i}= \begin{cases}1 & \text { for } i=1 \\ \min \left[1, k x_{i-1}\right] & \text { for } i>1\end{cases}
$$

It can be easily verified that $T \in \mathscr{L}(k, X)$. Now notice that for an arbitrary sequence $x \in X$ there exists $i$ such that $x_{i-1} \geqq 1 / k$ and $x_{i} \leqq$ $1 / k$. Hence $(T x)_{i}=1$ and

$$
\|T x-x\| \geqq\left|(T x)_{i}-x_{i}\right|=1-x_{i} \geqq 1-\frac{1}{k} .
$$

So in this case we also have

$$
\varphi_{c_{0}}(k)=1-\frac{1}{k}
$$

Some general properties of $\varphi_{B}(k)$. Before proving that the evaluation given above is not exact in Hilbert space let us consider some general properties of $\varphi_{B}(k)$.

Let $B$ be a fixed Banach space and let $X \in \mathfrak{M}_{B}, T \in \mathscr{L}(k, X)$. Then the mapping

$$
T_{\alpha}=(1-\alpha) \mathscr{I}+\alpha T
$$

where $\alpha \in\langle 0,1\rangle$ and $\mathscr{F}$ is identity transformation, belongs to $\mathscr{L}(1-$ $\alpha+\alpha k, X)$, and for arbitrary $x \in X$ we have

$$
\left\|x-T_{\alpha} x\right\|=\alpha\|x-T x\| \text {. }
$$

It follows that

$$
\varphi(1-\alpha+\alpha k, X) \geqq \alpha \varphi(k, X)
$$

and because the set $X$ and the transformation $T$ could be chosen so that

$$
\|x-T x\| \geqq r(X)(\varphi(k)-\varepsilon)
$$

then also we have

$$
\varphi(1-\alpha+\alpha k) \geqq \alpha \varphi(k) .
$$

This means that $\varphi(k)$ is concave with respect to the point $k=1$. This fact can be equivalently formulated as follows.

$$
\frac{\varphi(k)}{k-1} \geqq \frac{\varphi(l)}{l-1}
$$

for $1<k \leqq l$.

Because $\varphi(k) \leqq 1-1 / k$, the right derivative $\varphi^{\prime}(1)$ exists and 


$$
\varphi^{\prime}(1)=\lim _{k \rightarrow 1+} \frac{\varphi(k)}{k-1} \leqq 1
$$

Now take an arbitrary number $A>k$. For any fixed $x \in X$ we can define the transformation

$$
T_{x} y=\left(1-\frac{1}{A}\right) x+\frac{1}{A} T y .
$$

$T_{x} \in \mathscr{L}(k / A, X)$ and so it has a uniquely determined fixed point which we denote $F x$. Then we have

$$
F x=\left(1-\frac{1}{A}\right) x+\frac{1}{A} T F x .
$$

In view of

$$
\begin{aligned}
\|F x-F y\| & \leqq\left(1-\frac{1}{A}\right)\|x-y\|+\frac{1}{A}\|T F x-T F y\| \\
& \leqq\left(1-\frac{1}{A}\right)\|x-y\|+\frac{k}{A}\|F x-F y\|,
\end{aligned}
$$

the transformation $F$ belongs to $\mathscr{L}((A-1) /(A-k), X)$ and thus $T F \epsilon$ $\mathscr{L}(k(A-1) /(A-k), X)$. Moreover,

$$
\left(1-\frac{1}{A}\right)\|x-T F x\|=\|F x-T F x\|,
$$

so if $T$ and $X$ are chosen so that

$$
\|x-T x\| \geqq(\varphi(k)-\varepsilon) r(X)
$$

for $x \in X$, then

$$
x-T F x \| \geqq \frac{A}{A-1}(\varphi(k)-\varepsilon) r(X)
$$

and

$$
\varphi\left(k \frac{A-1}{A-k}, X\right) \geqq \frac{A}{A-1} \varphi(k(-\varepsilon) .
$$

Hence

$$
\varphi\left(k \frac{A-1}{A-k}\right) \geqq \frac{A}{A-1} \varphi(k) .
$$

The last inequality holds for arbitrary $A>k$. But for arbitrary $l>k$, $A$ can be chosen so that $k(A-1) /(A-k)=l$. After this substitution we get 


$$
\frac{l \varphi(l)}{l-1} \geqq \frac{k \varphi(k)}{k-1}
$$

for arbitrary $1<k \leqq l$. Hence

$$
\varphi^{\prime}(1)\left(1-\frac{1}{k}\right) \leqq \varphi(k) \leqq 1-\frac{1}{k}
$$

for all $k \geqq 1$.

The evaluations (2) and (3) show that $\varphi(k)=1-1 / k$ if and only if $\varphi^{\prime}(1)=1$; also $\varphi(k)=0$ if and only if $\varphi^{\prime}(1)=0$. For this reason $\varphi^{\prime}(1)$ can be considered as one of the characteristics of our problem.

The case of Hilbert space. In this section we shall deal with a Hilbert space $H$. In order to consider the function $\varphi(k)$ in $H$ we start with the following well known results (see e.g. [2]).

$1^{\circ}$. If $X \in \mathfrak{M}_{H}$, then $C(X)$ consists of exactly one point.

$2^{\circ}$. For arbitrary $x \in H$ there exists exactly one point $P x \in X$ such that

$$
\|x-P x\|=\min [\|x-y\|: y \in X] .
$$

$3^{\circ}$. The transformation $P$ is nonexpansive, i.e.,

$$
\|P x-P y\| \leqq\|x-y\|
$$

in $H$.

$4^{\circ}$. For arbitrary $y \in X$ and $x \in H$

$$
(P x-x, y-P x) \geqq 0,
$$

which in turn implies that

$$
\|y-x\| \geqq\|x-P x\| \text {. }
$$

The transformation $P$ will be called the matrical projection on $X$.

THEOREM 2. In Hilbert space, $\varphi(k)=\varphi\left(k, K_{1}\right)$.

Proof. Let $X \in \mathfrak{M}_{H}$ and let $z=C(X)$. The ball $K(z, r(X))$ covers $X$ and an arbitrary mapping $T \in \mathscr{L}_{k}(X)$ can be extended to all of $H$ by the formula $T^{*}=T P$, where $P$ is metrical projection on $X$, thus obtaining a transformation which belongs to $\mathscr{L}(k, K(z, r(X)))$. According to the properties of $P$ we have

$$
\left\|x-T^{*} x\right\|=\|x-T x\|
$$

for $x \in X$, and

$$
\left\|x-T^{*} x\right\| \geqq\|P x-T P x\|
$$


for $x \in K(z, r(X))-X$.

Hence

$$
\inf [\|x-T x\|: x \in X]=\inf \left[\left\|x-T^{*} x\right\|: x \in K(z, r(X))\right],
$$

and this implies that

$$
\varphi(k, X) \leqq \varphi(k, K(z, r(X)))=\varphi\left(k, K_{1}\right)
$$

for arbitrary $X \in \mathfrak{M}_{H}$, so therefore

$$
\varphi(k) \leqq \varphi\left(k, K_{1}\right) \text {. }
$$

The converse inequality is obvious and this completes the proof.

In view of Theorem 2 we need only deal with properties of $\mathscr{L}\left(k, K_{1}\right)$.

If $T \in \mathscr{L}\left(k, K_{1}\right)$ the for arbitrary $x \in K_{1}$ the transformation

$$
T_{x} y=\left(1-\frac{1}{k}\right) x+\frac{1}{k} T y
$$

belongs to $\mathscr{L}\left(1, K_{1}\right)$ and according to Browder's theorem [1] it has at least one fixed point. Let us choose one and denote it by $F x$. Thus we have

$$
F x=\left(1-\frac{1}{k}\right) x+\frac{1}{k} T F x
$$

or equivalently

$$
F x-T F x=(k-1)(x-F x)
$$

from which we get

$$
\inf \left[\|x-F x\|: x \in K_{1}\right] \geqq \frac{1}{k-1} \inf \left[\|x-T x\|: x \in K_{1}\right] \cdot
$$

Moreover, the following holds.

THEOREM 3. The transformation $F$ defined in (5) is monotone, and

$$
2(F x-F y, x-y) \geqq\left(1-\frac{1}{k}\right)\|x-y\|^{2}
$$

for $x, y \in K_{1}$.

Proof. Let $x, y \in K_{1}$. Then we have

$$
\begin{aligned}
& T F x=k F x-(k-1) x \\
& T E y=k F y-(k-1) y
\end{aligned}
$$


and

$$
\begin{aligned}
k^{2}\|F x-F y\|^{2} \geqq\|T F x-T F y\|^{2}= & k^{2}\|F x-F y\|^{2} \\
& -2(k-1) k(F x-F y, x-y) \\
& +(k-1)^{2}\|x-y\|^{2} .
\end{aligned}
$$

from which (6) follows.

THEOREM 4. In Hilbert space,

$$
\varphi(k) \leqq\left(1-\frac{1}{k}\right) \sqrt{\frac{k}{k+1}} .
$$

Proof. Let $T \in \mathscr{L}\left(k, K_{1}\right)$ be such that

$$
\|x-T x\| \geqq \varphi(k)-\varepsilon
$$

for all $x \in K_{1}$, and let $F$ be defined as above. Now we have

$$
\begin{aligned}
1 \geqq\left\|T F^{2} \theta\right\|^{2} & =\left\|T F^{2} \theta-F \theta+F \theta\right\|^{2} \\
& =\left\|T F^{2} \theta-F \theta\right\|^{2}+2\left(T F^{2} \theta-F \theta, F \theta-\theta\right)+\|F \theta-\theta\|^{2} \\
& \geqq k^{2}\left\|F^{2} \theta-F \theta\right\|^{2}+2 k\left(F^{2} \theta-F \theta, F \theta-\theta\right)+\|F \theta-\theta\|^{2} \\
& \geqq k^{2}\left\|F^{2} \theta-F \theta\right\|^{2}+k\|F \theta-\theta\|^{2} \\
& =\frac{k^{2}}{(k-1)^{2}}\left\|F^{2} \theta-T F^{2} \theta\right\|^{2}+\frac{k}{(k-1)^{2}}\|F \theta-T F \theta\|^{2} \\
& =\frac{k^{2}+k}{(k-1)^{2}}(\varphi(k)-\varepsilon)^{2} .
\end{aligned}
$$

Hence

$$
\varphi(k)-\varepsilon \leqq\left(1-\frac{1}{k}\right) \sqrt{\frac{k}{k+1}}
$$

which completes the proof.

CoRollary. In Hilbert space, $\varphi^{\prime}(1) \leqq 1 / \sqrt{2}$.

This theorem shows that infinite dimensional Banach spaces differ with respect to the minimal distance problem. Probably the evaluation (7) is not exact, and in fact it is not even known whether $\varphi(k)>$ 0 in $H$. In the next section we shall formulate an equivalent form of this latter question.

The retraction problem. We shall still deal here with the transformations of unit ball in Hilbert space. If $T: K_{1} \rightarrow K_{1}$ is a fixed point free continuous mapping, then the transformation 


$$
R x=x+t(x) \cdot u(x)
$$

where

$$
u(x)=\frac{x-T x}{\|x-T x\|}
$$

and

$$
t(x)=-(x, u(x))+\sqrt{1-\|x\|^{2}+(x, u(x))^{2}}
$$

is a retraction of $K_{1}$ onto $S_{1}$. It is well known that Brouwer's fixed point theorem is equivalent to the nonexistence of a retraction of a finite dimensional ball onto its boundary. The following theorem may be viewed as a counterpart of this fact.

THEOREM 5. In Hilbert space, $\varphi(k)>0$ for $k>1$ if and only if there exists a Lipschitzian retraction of $K_{1}$ onto $S_{1}$.

Proof. Suppose $T \in \mathscr{L}\left(k, K_{1}\right)$ is such that inf $\|x-T x\|=a>0$. Construct the retraction $R$ by formula (8). It can be easily verified that the functions $u(x),(x, u(x))$ are also Lipschitzian. The function $t(x)$ is Lipschitzian if $1-\|x\|^{2}+(x, u(x))^{2}$ is bounded from below by a positive number. But this holds if $(x, u(x))$ is bounded from below by a positive number for $\|x\|=1$. For $\|x\|=1$ we have

$$
\begin{aligned}
1 \geqq\|T x\|^{2} & =\|x-(x-T x)\|^{2}=\left\|x^{2}\right\|-2(x, x-T x)+\|T x-x\|^{2} \\
& \geqq 1-2(x, x-T x)+a\|x-T x\|,
\end{aligned}
$$

so

$$
(x, u(x)) \geqq \frac{a}{2}>0
$$

It follows that $R$ is Lipschitzian.

On the other hand, if $R \in \mathscr{L}\left(k, K_{1}\right)$ is a Lipschitzian retraction of $K_{1}$ onto $S_{1}$ then consider the transformation $T=-R$. Let $a=$ inf $\|x-T x\|$. If $a$ were 0 , then for arbitrary $\varepsilon>0$ there would exits $x \in K_{1}$ such that $\|x-T x\|<\varepsilon$. But $\left\|T x-T^{2} x\right\|=2$, so

$$
k \geqq \frac{\left\|T^{2} x-T x\right\|}{\|T x-x\|} \geqq \frac{2}{\varepsilon}
$$

and since $\varepsilon>0$ is arbitrary this is a contradiction. Thus $a>0$ and $\varphi(k)>0$.

Using this theorem we can show that the evaluation from below of $\varphi(k)$ given by (4) is also not exact if $\varphi(k)>0$. 
THEOREM 6. If $\varphi(k)>0$ for $k>1$, then $\lim _{k \rightarrow \infty} \varphi(k)=1$

Proof. If $\varphi(k)>0, k>1$, then there exists a Lipschitzian retraction $R$ of $K_{1}$ onto $S_{1}$. Hence there also exists a Lipschitzian retraction $R_{\varepsilon}$ of $K_{\varepsilon}$ onto $S_{\varepsilon}$. Thus the transformation

$$
T x=\left\{\begin{array}{lll}
-\frac{R_{\varepsilon} x}{\varepsilon} & \text { for } & \|x\| \leqq \varepsilon \\
-\frac{x}{\|x\|} & \text { for } & \|x\|>\varepsilon
\end{array}\right.
$$

belongs to $\mathscr{L}\left(K_{1}\right)$, and inf $\|x-T x\| \geqq 1-\varepsilon$, completing the proof.

REMARK. If $R_{\varepsilon}$ is only continuous, then $T$ is also continuous and $\|x-T x\| \geqq 1-\varepsilon$. However, it can be easily proved that there is no continuous self mapping of $K_{1}$ such that $\|x-T x\| \geqq 1$.

THEOREM 7. If there exists a retraction $R: K_{1} \rightarrow S_{1}$ such that $R \in$ $\mathscr{L}\left(k, K_{1}\right)$, then

$$
k \varphi(k) \geqq \pi
$$

Proof. The transformation $T=-R$ belongs to $\mathscr{L}\left(k, K_{1}\right)$ so for $\varepsilon>0$ there exists $x \in K_{1}$ such that $\|x-T x\| \leqq \varphi(k)+\varepsilon$. The segment $[x, T x]=[y: y=\alpha x+(1-\alpha) T x, \alpha \in\langle 0,1\rangle]$ is mapped by $T$ onto a curve lying on $S_{1}$ and joining the two antipodal points $T x$ and $T^{2} x=$ $-T x$. It has been proved by J. J. Schäffer [6] that the length of such a curve have has to be at least $\pi$. So it is only a slight technicality to prove that

$$
k \geqq \frac{L}{\|x-T x\|} \geqq \frac{\pi}{\varphi(k)+\varepsilon} .
$$

COROLLARY. According to (7), $k$ satisfies

$$
(k-1) \sqrt{\frac{k}{k+1}} \geqq \pi \text {. }
$$

Some unsolved problems. The preceding considerations give rise to several questions and problems, among them the following:

$1^{\circ}$. Does there exist an infinite dimensional Banach space (e.g. Hilbert space) for which $\varphi(k)=0$ ?

$2^{\circ}$. What is a characterization of spaces for which $\varphi(k)=1-1 / k$ ? Do nonreflexive spaces have this property?

$3^{\circ}$. Is it true that if $\varphi(k)>0$ then $\lim _{k \rightarrow \infty} \varphi(k)=1$ in an arbitrary space?

$4^{\circ}$. If the answer of problem $1^{\circ}$ is negative, what is a strict 
evaluation for $\varphi(k)$ in Hilbert space?

$5^{\circ}$. Is it true that for an arbitrary Banach space $B, \varphi_{H}(k) \leqq$ $\varphi_{B}(k) ?$

$6^{\circ}$. What is the infimum of those numbers $a$ such that there exists Banach space $B$ with $\varphi_{B}^{\prime}(1)=a$ ? Is it $\varphi_{H}^{\prime}(1)$ ?

Some of these problems (notably $1^{\circ}$ ) appear to be quite difficult, while others appear more accessible. In particular, it is our opinion that the answers to $5^{\circ}$ and $6^{\circ}$ are affirmative.

\section{REFERENCES}

1. F. E. Browder, Nonexpansive nonlinear operators in a Banach space, Proc. Not. Acad. Sci., USA 54 (1965), 1041-1044.

2. W. Cheney and A. A. Goldstein, Proximity maps for convex sets, Proc. Amer. Math. Soc., 10 (1959), 448-450.

3. D. Göhde, Zum prinzip der kontraktiven Abbildung, Math. Nachr., 30 (1965), 251258.

4. W. A. Kirk, A fixed point theorem for mappings which do not increase distances, Amer. Math. Monthly, 72 (1965), 1004-1006.

5. Z. Opial, Lecture notes on nonexpansive and monotone mappings in Banach space, Brown University, 1967.

6. J. J. Schäffer, Inner diameter, perimeter and girth of spheres, Math. Ann., 133 (1967), 59-82.

Received November 23, 1971. Research supported by Kosciuszko Foundation while author was at the University of Iowa. I would like to express my thanks to Professor W. A. Kirk who gave me his assistance while preparing this paper.

Maria CURIe Sklodowska University, Poland

AND

UNIVERSITY OF IOWA 



\section{PACIFIC JOURNAL OF MATHEMATICS}

EDITORS

\author{
H. SAMELSON \\ Stanford University \\ Stanford, California 94305 \\ C. R. HOBBY \\ University of Washington \\ Seattle, Washington 98105
}

\author{
J. DUGUNDJI \\ Department of Mathematics \\ University of Southern California \\ Los Angeles, California 90007 \\ RICHARD ARENS \\ University of California \\ Los Angeles, California 90024
}

\section{ASSOCIATE EDITORS}

E. F. BECKENBACH

B. H. NeUManN

F. WOLF

K. YosHIDA

\section{SUPPORTING INSTITUTIONS}

UNIVERSITY OF BRITISH COLUMBIA

CALIFORNIA INSTITUTE OF TECHNOLOGY

UNIVERSITY OF CALIFORNIA

MONTANA STATE UNIVERSITY

UNIVERSITY OF NEVADA

NEW MEXICO STATE UNIVERSITY

OREGON STATE UNIVERSITY

UNIVERSITY OF OREGON

OSAKA UNIVERSITY
UNIVERSITY OF SOUTHERN CALIFORNIA

STANFORD UNIVERSITY

UNIVERSITY OF TOKYO

UNIVERSITY OF UTAH

WASHINGTON STATE UNIVERSITY UNIVERSITY OF WASHINGTON

AMERICAN MATHEMATICAL SOCIETY NAVAL WEAPONS CENTER 


\section{Pacific Journal of Mathematics}

\section{Vol. 45, No. $1 \quad$ September, 1973}

William George Bade, Complementation problems for the Baire classes .......... 1

Ian Douglas Brown, Representation of finitely generated nilpotent groups ........ 13

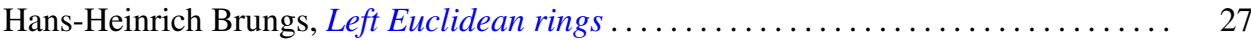

Victor P. Camillo and John Cozzens, A theorem on Noetherian hereditary rings ..... 35

James Cecil Cantrell, Codimension one embeddings of manifolds with locally flat

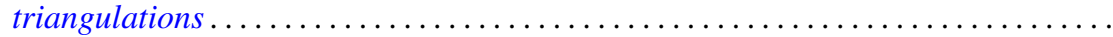

L. Carlitz, Enumeration of up-down permutations by number of rises . . . . . . . . . .

Thomas Ashland Chapman, Surgery and handle straightening in Hilbert cube

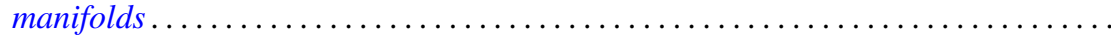

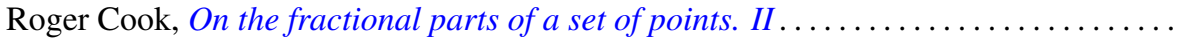

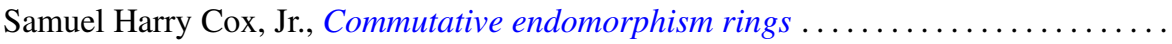

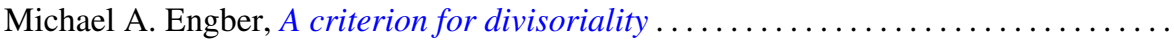

Carl Clifton Faith, When are proper cyclics injective . . . . . . . . . . . . . . 97

David Finkel, Local control and factorization of the focal subgroup . . . . . . . . . 113

Theodore William Gamelin and John Brady Garnett, Bounded approximation by

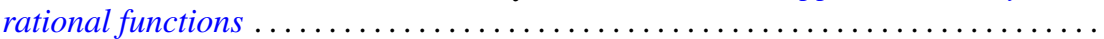

Kazimierz Goebel, On the minimal displacement of points under Lipschitzian

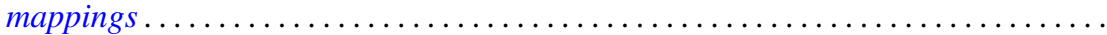

Frederick Paul Greenleaf and Martin Allen Moskowitz, Cyclic vectors for representations associated with positive definite measures: nonseparable

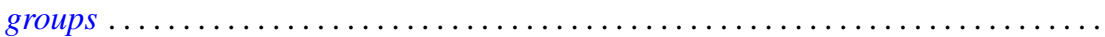

Thomas Guy Hallam and Nelson Onuchic, Asymptotic relations between perturbed linear systems of ordinary differential equations .

David Kent Harrison and Hoyt D. Warner, Infinite primes of fields and completions. .

James Michael Hornell, Divisorial complete intersections . ......

Jan W. Jaworowski, Equivariant extensions of maps ..............

John Jobe, Dendrites, dimension, and the inverse arc function .. .

Gerald William Johnson and David Lee Skoug, Feynman integrals of non-factorable

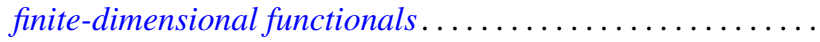

Dong S. Kim, A boundary for the algebras of bounded holomorphic functions ...... 269

Abel Klein, Renormalized products of the generalized free field and its derivatives ... 275

Joseph Michael Lambert, Simultaneous approximation and interpolation in $L_{1}$ and

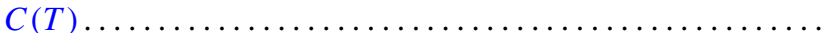

Kelly Denis McKennon, Multipliers of type $(p, p)$ and multipliers of the group

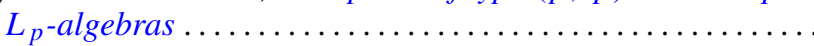

William Charles Nemitz and Thomas Paul Whaley, Varieties of implicative

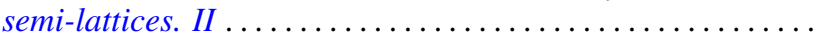

Donald Steven Passman, Some isolated subsets of infinite solvable

Norma Mary Piacun and Li Pi Su, Wallman compactifications on E-completely

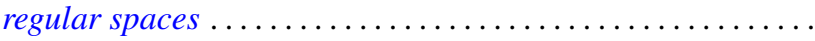

Jack Ray Porter and Charles I. Votaw, $S(\alpha)$ spaces and regular Hausdorff extensions....

Gary Sampson, Two-sided $L_{p}$ estimates of convolution transforms .

Ralph Edwin Showalter, Equations with operators forming a rig
Raymond Earl Smithson, Fixed points in partially ordered sets .

Victor Snaith and John James Ucci, Three remarks on symmetric products and

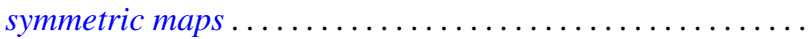

